This item was submitted to Loughborough's Research Repository by the author.

Items in Figshare are protected by copyright, with all rights reserved, unless otherwise indicated.

\title{
Training induced changes in quadriceps activation during maximal eccentric contractions
}

PLEASE CITE THE PUBLISHED VERSION

https://doi.org/10.1016/j.jbiomech.2018.03.025

PUBLISHER

(C) Elsevier

VERSION

AM (Accepted Manuscript)

\section{PUBLISHER STATEMENT}

This work is made available according to the conditions of the Creative Commons Attribution-NonCommercialNoDerivatives 4.0 International (CC BY-NC-ND 4.0) licence. Full details of this licence are available at: https://creativecommons.org/licenses/by-nc-nd/4.0/

\section{LICENCE}

CC BY-NC-ND 4.0

\section{REPOSITORY RECORD}

Voukelatos, Dimitrios, Mathew Kirkland, and Matthew T.G. Pain. 2019. "Training Induced Changes in Quadriceps Activation During Maximal Eccentric Contractions”. figshare. https://hdl.handle.net/2134/32880. 
1 Training induced changes in quadriceps activation during maximal eccentric 2 contractions.

3 Dimitrios Voukelatos ${ }^{1}$, Mathew Kirkland ${ }^{1,2}$, and Matthew TG Pain ${ }^{1}$

$4{ }^{1}$ School of Sport, Exercise and Health Sciences, Loughborough University, LE11 3TU, UK.

$5 \quad{ }^{2}$ St Peter's College, Johannesburg, South Africa

6

7

8 Submitted as Original Article

9 Corresponding author: Matthew TG Pain

10 School of Sport, Exercise and Health Sciences

11 Loughborough University

12 Loughborough LE11 3TU

13 UK

14 Email: m.t.g.pain@lboro.ac.uk

15 Tel. no: +44 (0)1509 226327

16

17 Dimitrios Voukelatos

18 School of Sport, Exercise and Health Sciences

19 Loughborough University

20 Loughborough LE11 3TU

$21 \quad \mathrm{UK}$

22 Email: D.Voukelatos@1boro.ac.uk

23 Tel. no: +44 (0)1509 226322

24

25 Mathew Kirkland

26 St Peter's College, Johannesburg, South Africa

27 Email: kirklandm@stpeterscollege.co.za 


\section{Abstract}

29 Despite full voluntary effort, neuromuscular activation of the quadriceps group of muscles appears inhibited during eccentric contractions. A nerve stimulation protocol during dynamic contractions of the quadriceps was developed that employed triplets of supramaximal pulses to assess suppressed eccentric activation. Subsequently the effects of a short training intervention, performed on a dynamometer, on eccentric strength output and neural inhibition were examined. Torque-angular velocity $(\mathrm{T}-\omega)$ and experimental voluntary neural driveangular velocity (\%VA- $\omega$; \%VA, obtained via the interpolated twitch technique) datasets, were obtained from pre- and post-training testing sessions. Non-linear regression fits of a seven parameter torque function and of a $3^{\text {rd }}$ degree polynomial were performed on the preand post-training T- $\omega$ and $\%$ VA- $\omega$ datasets respectively. T-test showed a significant $(p<$ 0.05) increase in the overall torque output post-training for the group, with three out of the six subjects demonstrating a significant $(p<0.05)$ increase in the torque output across the range of angular velocities as shown by the extra-sum-of-squares F-test. A significant increase $(p<$ $0.05)$ in the \%VA post-training was also observed as well as a reduction in the plateauing of the torque output during fast eccentric contractions.

Keywords: Neural inhibition, muscular contraction, stimulation, training. 
49 The maximal force generating capacity of a muscle is a function of its velocity and length.

During in vitro studies researchers have repeatedly shown isolated muscle fibres stretched under maximal tetanic conditions produce a force that is 1.5 to 1.9 times higher than maximal isometric force (Katz, 1939; Délèze, 1961; Edman et al., 1978; Edman, 1988). However, in vivo measurements of the torque-velocity profile during maximum voluntary contractions (MVC) show either little difference between isometric and eccentric torque across increasing angular velocities (Westing, 1988), or a tendency to decline with increasing velocity (Westing et al., 1990; Dudley et al., 1990; Pain \& Forrester, 2009; Forrester \& Pain, 2010). EMG studies have shown a 10-30\% decrease in the neural drive of the quadriceps under fast eccentric MVC contractions (Westing et al., 1991; Enoka, 1997; Paillard et al., 2005). It has been proposed that this apparent reduction in neural drive could be due to the existence of a neural tension-limiting mechanism that only becomes active during maximal load contractions of skeletal muscle (Westing et al., 1990; Westing et al., 1991). Pain and Forrester (2009) used normalized wavelet transformed EMG to calculate EMG-corrected maximal voluntary torques (MVT) from a wide range of eccentric and concentric contractions of the knee extensors. They arrived at a peak eccentric to isometric torque ratio $\left(\mathrm{T}_{\mathrm{ecc}} / \mathrm{T}_{0}\right)$ of 1.6.

Dudley et al. (1990) used sub-maximal transcutaneous electrical muscle stimulation (40-60\% of MVT) to produce a torque-velocity profile for the knee extensors that was closer to the in vitro tetanic profile; $\mathrm{T}_{\mathrm{ecd}} / \mathrm{T}_{0}$ of 1.4 and did not drop off at higher lengthening velocities. Westing et al. (1990) also used transcutaneous electrical muscle stimulation, in isolation and superposed on MVC, and although these authors attempted to obtain maximal activation levels using both methods the level of stimulation was subjectively limited between subjects based on their pain thresholds. They found that superposed stimulation increased eccentric 
MVT by $24 \%$ from MVC alone at $360 \%$. They obtained a $\mathrm{T}_{\mathrm{ecc}} / \mathrm{T}_{0}$ of 1.33 for stimulated

74

94 The results of Amiridis et al. (1996) suggest that the MVC and stimulated torque-velocity only, but 1.23 for superposed stimulation. For the latter the absolute torque values were higher and this was seen as a good indicator of the tension limiting mechanism. Amiridis et al. (1996) also used this superposition method and found similar results to Westing et al. (1990) for untrained subjects (torque with stimulation was 25\% higher than MVT alone, and $\mathrm{T}_{\mathrm{ecd}} / \mathrm{T}_{0}$ was 1.23 for MVC plus stimulation), but little eccentric increase for trained athletes when superposed electrical stimulation was used. For the athletes in the study of Amiridis et al. (1996) $\mathrm{T}_{\mathrm{ec}} / \mathrm{T}_{0}$ was 1.22 for superposed stimulation. More recently Pain et al. (2013) used sub-maximal transcutaneous muscle stimulation, but with a wider range of velocities than previously used, to obtain a $\mathrm{T}_{\mathrm{ecc}} / \mathrm{T}_{0}$ of 1.7 for both the quadriceps and hamstrings. In these studies lower absolute eccentric torque is associated with higher $\mathrm{T}_{\mathrm{ecc}} / \mathrm{T}_{0}$ ratios and is supportive of the tension limiting hypothesis.

The aforementioned studies have all used muscle stimulation which can cause rapid fatigue and discomfort and also reduces concentric torque values compared to MVT values. Transcutaneous stimulation of the femoral nerve is an alternative method for stimulating the quadriceps muscles, and has been used repeatedly in studies utilising the interpolated twitch technique (ITT) during isometric and slow dynamic contractions and in maximal rate of force development studies using octets (Deutekom et al., 2000; de Ruiter et al., 2004; Folland et al., 2014; Beltman et al., 2004). However, there does not appear to be any literature on repeated nerve stimulation during fast eccentric contractions and its effect on neuromuscular activation.

profiles may depend upon the fitness level of subjects. Therefore, it can be hypothesised that specific strength training could induce a reduction in the inhibitive action and a number of studies tested that hypothesis using various training programmes. These, however, were 
98 either performed using free weights (Aagaard et al., 2000), focused on the concentric phase 99 of muscular contraction only (Caiozzo et al., 1981), or the aim was to establish 100 training-induced physiological changes of the contracting muscles (Coyle et al., 1981; 101 Aagaard et al., 2001). Spurway et al. (2000) performed a 6 week knee extension training 102 protocol with one leg concentric and one leg eccentric and surmised from their results that 103 eccentric strength was increased primarily from decreased inhibition. However, no measures 104 of neural activity were taken and morphological changes would also likely have started.

105 Furthermore, attempts to improve the force output during maximal voluntary eccentric 106 contractions by following a strictly isovelocity strength training protocol have given 107 contradictory results (Higbie et al., 1996; Seger \& Thorstensson, 2005).

108 The aims of this study were: a) to develop a nerve stimulation protocol during dynamic 109 contractions without causing excessive discomfort or injury in order to examine suppressed 110 eccentric activation and b) to investigate whether performing a high velocity strength training 111 protocol using eccentric-concentric cycles on an isovelocity dynamometer would lead to a 112 decrease in the inhibitive action of the neural factors and an increase in torque output during 113 fast eccentric maximal voluntary contractions. The training protocol was specifically geared 114 to high velocity eccentric/concentric training on an isovelocity dynamometer over a period of 1153 weeks to limit adaptations to predominately neural changes (Corriander \& Tesch, 1990). It 116 was hypothesized that at the end of the training cycle subjects would exhibit significantly 117 higher torque outputs and a reduction in neural inhibition. 
119 Two similar groups of male volunteers, $(n=9$ and $n=6)$, who had not previously engaged in 120 any systematic form of strength training or high level sports practice, were recruited for the 121 study (mean \pm standard deviation: age $26.3 \pm 2.7$ years, body mass $72.9 \pm 11.7 \mathrm{~kg}$, height, $122172.2 \pm 8.4 \mathrm{~cm}$;). They all gave written, informed consent and the study was conducted in accordance with the approval given by the Loughborough University Ethical Advisory Committee. The study was divided into two phases to address aims (a) and (b) above.

Phase 1.

The minimum required sample size was determined by performing a power analysis on the MVC and superimposed eccentric torque values reported by Westing et al., (1990). The analysis showed that a minimum sample size of four was required to achieve a power value of 0.8 and $p<0.05$. To account for drop out a total of nine subjects took part in this phase of the study and data collection finished when six had completed the protocol. As this protocol was painful for some subjects, and pain was associated with an increased risk of injury, the 132 subject numbers were kept minimal for ethical considerations, and two more than the minimum completed testing in case of later issues with data. Testing took place on an isovelocity dynamometer with built-in gravitational torque correction (Con-Trex, CMV AG, Switzerland) over three sessions. In each session subjects were seated on the dynamometer with their dominant leg strapped tightly to the unpadded crank arm directly above the ankle

137 joint using a protective moulded plastic shin guard. The anterior hip angle was set at $100^{\circ}$ 138 (seat was set at $80^{\circ}$ incline). To minimise differences between the crank and joint kinematics, the rotational axis of the crank arm was aligned with the centre of the knee joint during near-maximal efforts. 
141 Dynamometer and stimulator data were recorded simultaneously at $512 \mathrm{~Hz}$ with Spike2

142 software (Spike 2, CED, Cambridge, UK). The dynamometer data were filtered at $8 \mathrm{~Hz}$

143 using a low-pass fourth order Butterworth filter. Knee joint angles were measured with a

144 mechanical goniometer during four isometric trials and the instantaneous crank arm angle

145 was converted to joint angle using a linear regression equation (Pain \& Forrester, 2009). For

146 each dynamic trial the maximum eccentric and concentric isovelocity phases were identified

147 and the isovelocity plateau was defined as the region where the angular velocity was within

$1485 \%$ of the peak value.

149 Each session was initiated with a standardized warm up protocol. Session 1 was a 150 familiarisation session where subjects performed one maximal MVC at crank angles of $15^{\circ}$ 151 through to $75^{\circ}$ in $15^{\circ}$ steps (with $0^{\circ}$ corresponding to full extension) and a number of MVC 152 and electrically stimulated dynamic (eccentric-concentric) contractions at 50, 200 and $350 \%$ s.

153 The optimum angle of peak torque was determined by fitting a quadratic to the torque-angle dataset obtained from the isometric MVCs. During the second session maximum, eccentricconcentric contractions were performed at: 50,200 and $350 \%$ s, according to the protocol of

156 Yeadon et al. (2006) with two-minute rest intervals between trials. Once MVCs were 157 completed subjects performed one stimulated trial at each isovelocity to further familiarise themselves with the sensation. Subsequently, optimum peak torque angles per isovelocity were determined for each subject as well as the time lapse between onset and effect of stimulation in order for the latter to coincide with the optimum angle. The onset 161 of stimulation varied with angular velocity and acceleration (Figure 1). However, the 162 changing width of stimulation twitch response with angular velocity (Gandevia et al., 1998) was not accounted for. In the third session subjects performed one MVC and one supramaximal stimulation trial at each isovelocity and each contraction mode and the respective peak torque values were recorded and used in the subsequent analysis. 
Electrical stimulation. Transcutaneous electrical stimulation of the quadriceps was achieved using a stimulator (DS7AH, Digitimer Ltd., UK) controlled by Spike 2 software. Two electrodes, a ball probe cathode of $10 \mathrm{~mm}$ in diameter, and a rectangular anode (90x50 mm) both coated with a thin layer of conductive gel were placed at the femoral nerve and the 170 gluteal fold respectively (Tillin et al., 2011). The individual stimulation intensity was determined by sending single rectangular pulses $(0.2 \mathrm{~ms})$ of increasing strength starting from a current intensity of $30 \mathrm{~mA}$, in $30 \mathrm{~mA}$ steps, until the twitch response plateaued. A supramaximal stimulation level was set at $20 \%$ above this intensity. In the first session a singlet supramaximal pulse was sent through the femoral nerve in order to 175 gradually familiarise the subjects to electrical stimulation, however, this became a triplet in subsequent sessions. The pulses were timed to coincide with optimum knee angle.

177 A $2 \times 4$ repeated measures ANOVA was performed in order to determine the effects of 178 stimulus (MVC vs STIM) and velocity on the torque values. Effect sizes were calculated and 179 subsequently used in a second power analysis to determine the minimum sample size for the 180 training part of the study.

\section{Phase 2}

182 Having established that triplets would not drive eccentric values high enough to reach 183 theoretical $\mathrm{T}_{\mathrm{ecc}} / \mathrm{T}_{0}$ values the use of doublet stimulation was chosen for the ITT, since it has been shown that the method is not sensitive to the number of pulses used, allowing the measurement of reduced voluntary activation but with less discomfort (Behm et al., 1996; Folland \& Williams, 2007). This would help mitigate the risk of losing subjects in the latter

187 stages of the testing protocol when replacements would not be possible. Power analysis based on Phase 1 showed that a minimum sample size of $n=5$ was required to achieve a power value of 0.8 and $p<0.05$. Six new subjects were recruited in this phase of the study. 
190 Phase 2 consisted of eleven sessions, a familiarisation session that followed the 191 familiarisation protocol of Phase 1, eight training sessions and two testing sessions that took 192 place pre- and post-training respectively. Training took place over a 3-week period. Sessions 193 lasted no more than 30 minutes, where subjects performed up to 10 sets of dynamic eccentric194 concentric knee extension cycles at velocities ranging between 50 and $350 \%$ s. The number of cycles and velocities increased as subjects adapted. Since the intensity of the training could not be quantified by counting the number of repetitions and loads, sets were time-matched. Specifically, one eccentric-concentric cycle was performed at $50 \%$ and $100 \%$, two at $150 \%$, three at $250 \%$ s and four at $350 \%$. All training sessions were supervised by the investigators.

199 The testing protocol consisted of maximal voluntary and supramaximally 200 electrically stimulated isometric and dynamic contractions. The range of isometric 201 contractions was the same as in previous sessions but this time the dynamic contractions 202 were measured at 5 angular velocities: 50, 100, 150, 250 and 350\%s. During isometric contractions subjects performed one MVC and one stimulation contraction per joint angle. The same order was maintained during dynamic contractions. Electrical stimulation was achieved following the procedure described in Phase 1 with doublet pulses.

206 The percentage of voluntary activation (\%VA) of the quadriceps muscle was expressed by 207 the following formula:

where the superimposed twitch is the torque increment noted during a maximal contraction at 210 the time of stimulation and the control twitch is that evoked in the relaxed muscle (Shield \& 211 Zhou, 2004; Folland \& Williams, 2007). The torque increment was defined as follows. If torque was increasing in value prior to stimulation then the value of the torque in the absence 
214 onset, taking the corresponding extrapolated value and subtracting it from the peak twitch

215 torque, similar to Gandevia et al. (1998). If torque value was decreasing prior to stimulation,

216 and in order to avoid overestimating the torque increment, the last value prior to onset of

217 stimulation was subtracted from the peak twitch torque value, similar to Beltman et al. (2004)

218 (Figure 1).

219 In order to assess possible group changes in performance the torque vs. angular velocity (T$220 \omega)$ curves were plotted for every subject pre and post-training. These were numerically 221 integrated and the eccentric and concentric areas compared at group level using a one-tailed 222 paired t-test. A 2x2x6 repeated measures ANOVA (time x velocity x contraction mode) was also used to determine the effects of velocity and training on the neural inhibition during eccentric contractions. Due to difficulties in eliciting stimulated contractions at the predetermined angles during efforts at high isovelocities it was not possible to repeat the ttest comparison for the ITT dataset due to the small number of data points obtained.

T- $\omega$ and \%VA- $\omega$ data sets per subject were obtained in both testing sessions. The individual pre- and post-training T- $\omega$ data sets for each subject were statistically compared by performing a nonlinear regression fit of the 7-parameter MVT function defined in Forrester et al. (2011), first separately and subsequently to the combined pre and post-training data sets (Figure 2). The fits for each profile were statistically compared using the extra-sum-ofsquares F-test (Motulsky \& Christopoulos, 2004; Voukelatos \& Pain, 2015). The same statistical process was repeated for the $\%$ VA- $\omega$ data set by fitting a $3^{\text {rd }}$ degree polynomial to establish the training effect on voluntary activation (Figure 3 ).

Normal distribution was checked using a Shapiro-Wilk test of normality. Analysis of the

236 Con-Trex data was performed using Matlab (version 8.1, The MathWorks Inc., Natick, MA, USA) and statistical analysis was performed using SPSS (version 21, SPSS Inc., Chicago, 
238 Illinois, USA). The power analyses were performed using GPower (Erdfelder et al., 2009). 239 A statistical level of significance, $p<0.05$, was used throughout. Cohen's, $d$, was used as an 240 effect size for the t-tests considering 0.2, 0.5, 0.8 as small, medium and large effects. Effect 241 size for the factorial ANOVAs used the partial eta squared statistic, $\eta_{p}^{2}$, (Cohen, 1992). Data 242 are reported as mean \pm SD unless otherwise stated. 


\section{Results}

Phase1

The $2 \times 4$ factorial ANOVA showed that there was a significant main effect for stimulus $(F=$

245

246

247

248

249

250

251

252

253

254

255

256

257

258

259

260

261

262

263

264

265 The individual MVT fit to each subject's T- $\omega$ datasets (Figure 2) showed that 3 out of the 6

67, $\left.\eta_{p}^{2}=0.94\right)$. Contrasts between the baseline torque value recorded at $350 \%$ showed significant increase in torque outputs during stimulation contractions with respect to torque outputs from $200 \%$ and $50 \%$ s (Table 1 ).

[Table 1]

Phase 2

The comparison of the numerically integrated $\mathrm{T}-\omega$ plots using a paired t-test showed significant increase $(t=3.2, d=1.3)$ between pre and post-training data. There were significant increases in area under the T- $\omega$ curve post-training for both the eccentric section, $t$ $=2.0, d=0.82$ and the concentric section, $t=2.3, d=0.93$.

The $2 \times 2 \times 6$ factorial ANOVA revealed a significant main effect for time $\left(F=6.6, \eta_{p}^{2}=0.57\right)$ with overall post-training torque output being significantly higher than pre-training values (239 \pm 12 vs $261 \pm 15 \mathrm{Nm}$ for pre and post-training respectively). There was no significant time $\mathrm{x}$ velocity interaction. Contrasts were also performed comparing peak torque output from $0-250 \%$ s the baseline value of $350 \%$ s. Those revealed a significant increase in eccentric peak torque from $0-250 \%$ s to the baseline value of $350 \%$, relative to peak torque values at $150 \%$ s (Table 2).

[Table 2]

subjects had significantly higher torque output post-training (Table 3 ). When the MVT 
267 function was fitted to the pooled pre and post T- $\omega$ datasets of all subjects a significant 268 increase in torque output post-training was found at group level $(F=2.06, d=0.63)$.

269 Applying the extra-sum-of-squares F-Test (Figure 3) to the \%VA- $\omega$ datasets of each subject 270 individually revealed one subject with a significant difference in \%VA post-training (Table 271 3). However, the combined curve fit to the pooled pre and post-training $\%$ VA- $\omega$ datasets 272 showed a significant increase in the \%VA $(F=3.3, d=0.39)$.

273

274 [Table 3]

275

276 [Figure 1]

277

278 [Figure 2]

279

280 [Figure 3]

281

282

[Figure 4]

283

284 Discussion

285 The aim of the first phase was to develop a nerve stimulation protocol during dynamic 286 contractions in order to examine suppressed eccentric activation and was for the most part 287 successful. Subjects achieved significantly higher torque outputs during electrically 288 stimulated eccentric contractions of the quadriceps compared to the respective MVC values 289 (Table 1). Moreover the repeated measures ANOVA contrasts showed that triplet stimulation 290 successfully reduced the torque suppression in the eccentric region of the T- $\omega$ curve. At 
$350^{\circ} / \mathrm{s}$ the peak torque with stimulation superposed was $31 \%$ higher than that of MVC alone and this is greater than that seen in Westing et al. (1990) and Amiridis et al. (1996). In this study $\mathrm{T}_{\mathrm{ecc}} / \mathrm{T}_{0}$ was 1.24 during superposed nerve stimulation, which is the same as the 1.23 times found in both Westing et al. (1990) and Amiridis et al. (1996). The differences in the ratios of increased eccentric torque and $\mathrm{T}_{\mathrm{ecc}} / \mathrm{T}_{0}$ between this study and the previous ones are likely due to the low eccentric MVT values of the subjects in this study. A limitation of our method can be found in the accuracy of timing of the triplet stimulation, particularly at high velocity. Consequently, the peak STIM torque angle may not always coincide with the peak MVC torque angle. However, it is likely that VA is less susceptible to timing errors as during maximal effort trials (STIM or MVC) subjects are meant to be maximally active and therefore the twitch increment will still be relative to maximum effort. Another potential limitation of using triplet stimulation is the level of discomfort felt by subjects. This may also explain the lower values for MVT via a fear avoidance reduction of volitional effort over and above the potential neural inhibition (Button \& Behm, 2008). This was predominantly observed during isometric contractions where three of the six subjects recorded STIM values that were significantly lower than their respective MVC values. Given subject comments and that a typical twitch response can be seen that does not drive the torque value towards the MVC, this was likely due to increased whole body tension and degree of co-contraction of the antagonist (Figure 4).

310 At the end of the short term high velocity dynamometer training protocol subjects achieved a

311 significant increase in overall torque output during both concentric and eccentric 312 contractions, in agreement with our hypothesis. Regarding the effect of the training protocol 313 on neural activation and the action of the tension limiting mechanism, a significant increase 314 in the \%VA post-training was achieved, as well as a significant increase in the peak torque outputs, during eccentric contractions at $350 \%$ with respect to torque outputs from $150 \%$ s. 
316 These results are indicative of increased neuromuscular activation post-training and a 317 possible reduction in the inhibitive action of the tension limiting mechanism. These results 318 are in, at least partial, agreement with previous isovelocity training studies that also reported 319 significant increases in the torque output during eccentric/concentric contractions of the 320 quadriceps after isovelocity strengthening protocols (Caiozzo et al., 1981; Coyle et al., 1981; 321 Hortobàgyi et al., 1996; Higbie et al., 1996).

322

323

324

325

The current study also sought to address the nature of the underlying reason behind increased torque output post-training, and more specifically whether this was due to an increase in neuromuscular activation. The significant increase in the \%VA value post-training suggests an increase in the neuromuscular activation of the quadriceps muscle. This is in line with findings by Hortobàgyi et al. (1996), Higbie et al. (1996) and Aagaard et al. (2000) who reported increased iEMG activity of the quadriceps muscle post-training. However, since post-training increases in quadriceps cross-sectional area and number of type II fibres were also reported (Higbie et al., 1996; Hortobàgyi et al., 1996), it is not clear whether the observed increases in iEMG values were solely due to increased neuromuscular activation or also due to increased muscle hypertrophy. In the current study only 8 training sessions in three weeks took place, thus it is likely that the observed increase in the torque output posttraining can be attributed almost exclusively to neural factors, such as increased muscle neuromuscular activation, more efficient recruitment and decreased neural inhibition (Staron et al., 1994; Colliander \& Tesch, 1990).

Increased neuromuscular activation would manifest itself through a greater increase in torque output during eccentric compared to concentric contractions post-training and a reversal of the torque suppression during eccentrics at high velocities in vivo (Westing et al., 1990, 1991; Dudley et al., 1990; Webber \& Kriellaars, 1997; Seger \& Thorstensson, 2005). The observed torque increase in this study was not higher post-training during eccentric compared to 
concentric contractions. However, the results of the repeated measures ANOVA showed that

342 whereas the subjects' torque outputs tended to plateau at $150 \%$ s during eccentric contractions

343 pre-training they do not appear to do so post-training. This is possibly a significant finding as

344 it offers an indication that the neural inhibition may, indeed be reversible. At the same time it

345 must be noted that, unlike Phase 1, there was no clear increasing trend in eccentric peak

346 torque values with increasing angular velocities suggesting that some level of neural

347 inhibition was possibly still present post-training. If this is indeed the case then, a complete

348 reversal of neural inhibition may need longer periods of training to emerge if the inhibition is

349 present to act against overloading the musculoskeletal system. To safely increase eccentric

350 strength concomitant increases in resistance to loading of the tendons, bones, and other

351 structural tissues would be necessary and take longer to adapt.

352 Limitations of this study include the difficulty of eliciting consistent electrical pulses at high

353 isovelocities during stimulated contractions, the use of two different stimulation protocols

354 due to subject discomfort, and possible learning effects from the repeated use of the

355 dynamometer by the subjects. The change to using doublet ITT to look at voluntary

356 activation via twitch responses prevents some direct comparisons between Phase 1 and Phase

3572 results but still reflects the activation changes. The familiarization session protocol was

358 designed to minimize learning effects and their confounding influence (Madsen, 2006; Lund 359 et al., 2005) and should not be a major factor.

360 This is the first time triplet nerve stimulation has been used to assess eccentric suppression

361 during fast dynamic contractions and produced very similar results to muscle stimulation studies whilst also allowing the application of dynamic doublet ITT to eccentric and concentric knee extensions. Performing a short, strength training protocol, consisting of 8 training sessions, on an isovelocity dynamometer over a range of angular velocities produced notable increases in torque output for all velocities and types of contraction. This is 
366 attributed to an increase in muscle activation and, a decrease in the inhibitive action of the 367 tension-limiting mechanism observed during fast eccentric contractions of the quadriceps.

\section{Disclosure of conflict of interest}

369 There is no conflict of interest. 


\section{References}

372 Aagaard, P., Simonsen, E. B., Andersen, J. L., Magnusson, S. P., Dyhre-Poulsen, P., Halkjær373 Kristensen, J., 2000. Neural inhibition during maximal eccentric and concentric quadriceps contraction: effects of resistance training. Journal of Applied Physiology, 89, 2249-2257.

376

Aagaard, P., Andersen, J. L., Dyhre-Poulsen, P., Leffers, A. M., Wagner, A., Magnusson, S. P., Halkjær-Kristensen, J., \& Simonsen, E. B., 2001. A mechanism for increased contractile strength of human pennate muscle in response to strength training: changes in muscle architecture. Journal of Physiology, 534, 613-623.

381

Amiridis, I. G., Martin, A., Morlon, B., Martin, L., Cometti, G., Pousson, M., van Hoecke, J., 1996. Co-activation and tension-regulating phenomena during isokinetic knee extension in sedentary and highly skilled humans. European Journal of Applied Physiology, 73, 149156.

386

Behm, D. G., St-Pierre, D. M. M., Perez, D., 1996. Muscle inactivation: assessment of interpolated twitch technique. Journal of Applied Physiology, 81, 2267-2273.

389

390

Beltman, J. G. M., Sargeant, A. J., van Mechelen, W., de Haan, A. 2004. Voluntary activation 391 level and muscle fiber recruitment of human quadriceps during lengthening contractions. Journal of Applied Physiology, 97, 619-626.

Button, D. C., Behm, D. G., 2008. The effect of stimulus anticipation on the interpolated twitch technique. Journal of Sports Science and Medicine, 7, 520-524. 
396 Caiozzo, V. J., Perrine, J. J., Edgerton, V. R., 1981. Training-induced alterations of the in 397 vivo force-velocity relationship of human muscle. Journal of Applied Physiology, 51, 750$398 \quad 754$

399

400 Cohen, J., 1992. Statistical power analysis. Current Directions in Psychological Science, 1, $40198-101$.

402

403 Corriander, E., B., \& Tesch, P., A., 1990. Effects of eccentric and concentric muscle actions 404 in resistance training. Acta Physiologica Scandinavica, 140, 31-39.

405

406 Coyle, E. F., Feiring, D. C., Rotkis, T. C., Cote III, R. W., Roby, F. B., Lee, W., Wilmore, J. 407 H., 1981. Specificity of power improvements through slow and fast isokinetic training. $408 \quad$ Journal of Applied Physiology, 51, 1437-1442.

409

410 Délèze, J.B., 1961. The mechanical properties of the semitendinosus muscle at lengths 411 greater than its length in the body. Journal of Physiology, 158, 154-164.

412

413 de Ruiter, C. J., Kooistra, R. D., Paalman, M. L, de Haan, A., 2004. Initial phase of maximal 414 voluntary and electrically stimulated knee extension torque development at different knee 415 angles. Journal of Applied Physiology, 97, 1693-1701.

416

417 Deutekom, M., Beltman, J. G. M., de Ruiter, C. J., Koning, J. J., de Haan, A., 2000. No acute 418 effects of short-term creatine supplementation on muscle properties and sprint 419 performance. European Journal of Applied Physiology, 82, 223-229. 
Dudley, G. A., Harris, R. T., Duvoisin, M. R., Hather, B. M., Buchanan, P., 1990. Effect of voluntary vs. artificial activation on the relationship of muscle torque to speed. Journal of Applied Physiology, 69, 2215-2221.

Edman, K. A. P., Elzinga, G., Noble, M. I. M., 1978. Enhancement of mechanical performance by stretch during tetanic contractions of vertebrate skeletal muscle fibres. Journal of Physiology, 281, 139-155.

Edman, K. A. P., 1988. Double hyperbolic force-velocity relation in frog muscle fibers. Journal of Physiology, 404, 301-321.

Enoka R. M., 1997. Neural adaptations with chronic physical activity. Journal of Biomechanics, 30, 447-455.

Erdfelder, F. F., Buchner, A., Lang, A. G., 2009. Statistical power analyses using GPower 3.1: Tests for correlation and regression analyses. Behavior Research Methods, 41, 11491160.

Folland, J. P. \& Williams, A. G., 2007. Methodological issues with the interpolated twitch technique. Journal of Electromyography and Kinesiology, 17, 317-327.

Folland, J. P., Buckthorpe, M. W., Hannah, R., 2014. Human capacity for explosive force production: Neural and contractile determinants. Scandinavian Journal of Medicine and Science in Sports, 24, 894-906. 
446 Forrester, S. E. \& Pain, M.T.G., 2010. A combined muscle model and wavelet approach to

447 interpreting the surface EMG signals from maximal dynamic knee extensions. Journal of $448 \quad$ Applied Biomechanics, 26, 62-72.

449

450 Forrester, S. E., Yeadon, M. R., King, M. A., Pain, M.T.G., 2011. Comparing different $451 \quad$ approaches for determining joint torque parameters from isovelocity dynamometer 452 measurements. Journal of Biomechanics, 44, 955-961.

453

454 Gandevia. S. C., Herbert, R. D., Leeper, J. B., 1998. Voluntary activation of human elbow 455 flexor muscles during maximal eccentric contractions. Journal of Physiology, 512, 595$456 \quad 602$.

457 Hortobàgyi, T., Hill, J. P., Houmard, J. A., Fraser, D. D., Lambert, N. J., Israel, R. G., 1996. 458 Adaptive responses to muscle lengthening and shortening in humans. Journal of Applied $459 \quad$ Physiology, 80, 765-772.

460

461 Higbie, E. J., Cureton, K. J., Warren III, G. L., Prior, B. M., 1996. Effects of concentric and 462 eccentric training on muscle strength, cross-sectional area, and neural activation. Journal 463 of Applied Physiology, 81, 2173-2181.

464

465 Katz, B., 1939. The relation between force and speed in muscular contraction. Journal of 466 Physiology, 96, 45-64.

467

468 Lund, H., Søndergaard, K., Zachariassen, T., Christensen, R., Bülow, P., Henriksen, M., 469 Bliddal, H., 2005. Learning effect of isokinetic measurements in healthy subjects and 
reliability and comparability of Biodex and Lido dynamometers. Clinical Physiology and $471 \quad$ Functional Imaging, 25, 75-82.

472

473 Madsen, O. R., 1996. Torque, total work, power, torque acceleration energy and acceleration 474 time assessed on dynamometer: reliability of knee and elbow extensor and flexor strength 475 measurements. European journal of Applied Physiology, 74, 206-210.

476

477 Motulski, H., \& Christopoulos, A., 2004. Fitting Models to Biological Data using Linear and $478 \quad$ Nonlinear Regression. Oxford University Press, New York.

479

480 Paillard, T., Margnes, E., Maitre, J., Chaubet, V., Francois, Y., Gonzalec, G., Borel, L., 2005. $481 \quad$ Electrical stimulation superimposed onto voluntary muscular contraction. Sports Medicine, 35, 951-966.

483

484 Pain, M. T. G. \& Forrester, S. E., 2009. Predicting maximum eccentric strength from surface 485 EMG measurements. Journal of Biomechanics, 42, 1598-1603.

486

487 Pain, M. T. G., Young, F., Forrester, S. E., 2013. The torque-velocity relationship in large 488 human muscles: maximum voluntary versus electrically stimulated behaviour. Journal of 489 Biomechanics, 46, 645-650.

490

491 Place, N., Casartelli, N., Glatthorn, J. F., Maffiuletti, N. A., 2010. Comparison of quadriceps 492 inactivation between nerve and muscle stimulation. Muscle Nerve, 42, 894-900. 
Seger, J. Y., \& Thorstensson, A., 2005. Effects of eccentric versus concentric training on thigh muscle strength and EMG. International Journal of Sports Medicine, 26, 45-52.

496

Shield, A., \& Zhou, S., 2004. Assessing Voluntary Muscle Activation with the Twitch Interpolation Technique. Sports Medicine, 34, 253-267.

499

500 Spurway, N. C., Watson, H., McMillan, K., Connoly, G., 2000. The effect of strength training 501 quadriceps. European Journal of Applied Physiology, 82, 374-380.

503

504 Staron, R. S., Karapondo, D. L., Kraemer, W. J., Fry, A. C., Gordon, S. E., Falkel, J. E., 505 Hagerman, F. C., Hikida, R. S., 1994. Skeletal muscle adaptations during the early phase 506 of heavy-resistance training in men and women. Journal of Applied Physiology, 76, 1247$507 \quad 1255$.

508

509 Tillin, N. A., Pain, M. T. G., Folland, J. P., 2011. Short term unilateral resistance training 510 affects the agonist-antagonist but not the force-agonist activation relationship. Muscle $511 \quad$ Nerve, 43, 375-384.

512

513 Voukelatos, D., \& Pain, M. T. G., 2015. Modelling suppressed muscle activation by means of 514 an exponential sigmoid function: Validation and bounds. Journal of Biomechanics, $515 \quad 48,712-715$

516

517 Weber, S., \& Kriellaars, D., 1997. Neuromuscular factors contributing to in vivo eccentric 518 moment generation. Journal of Applied Physiology, 83, 40-45. 
520 Westing, S. H., 1988. Eccentric and concentric torque-velocity characteristics of the

521 quadriceps femoris in man. European Journal of Applied Physiology, 58, 100-104.

522

523 Westing, S. H., Seger, J. Y., Thorstensson, A., 1990. Effects of electrical stimulation on

524 eccentric and concentric torque -velocity relationships during knee extension in man. Acta

525 Physiologica Scandinavica, 140, 17-22.

526

527 Westing, S. H., Cresswell, A. G., Thorstensson, A., 1991. Muscle activation during maximal $528 \quad$ voluntary eccentric and concentric knee extension. European Journal of Applied

529 Physiology, 62, 104-108.

530

531 Yeadon, M. R., King, \& M. A., Wilson, C., 2006. Modelling the maximum voluntary joint 532 torque/angular velocity relationship in human movement. Journal of Biomechanics, 39, $533 \quad 476-482$. 
Table 1: Mean peak torque \pm SD values per isovelocity obtained for MVC and stimulated eccentric contractions during Phase 1. It is noted that contrary to the other isovelocities, the stimulated peak torque values were lower than the respective MVC values as some of the subjects were adversely affected by the intensity of the stimulus.

\begin{tabular}{ccc}
\hline $\boldsymbol{\omega}(\%)$ & MVC $(\mathbf{N m})$ & STIM $(\mathbf{N m})$ \\
\hline $\mathbf{0}$ & $284 \pm 22$ & $268 \pm 38$ \\
$\mathbf{5 0}$ & $266 \pm 15$ & $291 \pm 25$ \\
$\mathbf{2 0 0}$ & $257 \pm 32$ & $318 \pm 39$ \\
$\mathbf{3 5 0}$ & $254 \pm 24$ & $333 \pm 60$ \\
\hline
\end{tabular}

538

539

540 Table 2: Mean peak \pm SD torque values obtained at 0 to $\pm 350^{\circ} / \mathrm{s}$ during pre and post-training 541 sessions for both contraction modes.

\begin{tabular}{ccccc}
\hline $\boldsymbol{\omega}(\%)$ & Pre-Training & Torque (Nm) & Post-Training & Torque (Nm) \\
& ECC & CONC & ECC & CONC \\
\hline $\mathbf{5 0}$ & $240 \pm 32$ & $188 \pm 26$ & $265 \pm 46$ & $207 \pm 18$ \\
$\mathbf{1 0 0}$ & $251 \pm 39$ & $168 \pm 27$ & $254 \pm 33$ & $196 \pm 19$ \\
$\mathbf{1 5 0}$ & $245 \pm 27$ & $152 \pm 19$ & $257 * \pm 29$ & $169 \pm 19$ \\
$\mathbf{2 5 0}$ & $226 \pm 34$ & $128 \pm 19$ & $251 \pm 41$ & $140 \pm 12$ \\
$\mathbf{3 5 0}$ & $247 \pm 42$ & $109 \pm 20$ & $280 * \pm 46$ & $127 \pm 13$
\end{tabular}


543 Table 3: Results obtained from fitting the MVT torque function and a $3^{\text {rd }}$ degree polynomial

544 to the raw T- $\omega$ and $\% \mathrm{VA}-\omega$ data sets respectively. Individual comparisons showed that three

545 out of six subjects recorded significantly higher torque outputs and one subject exhibited

546 significantly higher neuromuscular activation post-training.
MVC fit
\%VA fit

F-ratio $^{\dagger}$ Cohen's d $\quad$ F-ratio $^{\dagger} \quad$ Cohen's d

$\begin{array}{lllll}\text { Subject } 1 & 0.92 & 0.18 & 1.35 & 0.75\end{array}$

$\begin{array}{lllll}\text { Subject } 2 & 5.91^{*} & 2.1 & 1.93 & 0.24\end{array}$

$\begin{array}{lllll}\text { Subject } 3 & 1.58 & 0.71 & 0.67 & 0.11\end{array}$

$\begin{array}{lllll}\text { Subject } 4 & 4.95^{*} & 1.45 & 4.2 * & 0.35\end{array}$

$\begin{array}{lllll}\text { Subject } 5 & 12.9^{*} & 3.8 & 3.8 & 0.86\end{array}$

$\begin{array}{lllll}\text { Subject } 6 & 2.62 & 0.81 & 0.94 & 0.32\end{array}$

$547 * 0<0.05$

$548 \dagger \mathrm{F}$ represents the ratio between the sum of the variances of the pre and post-training MVC /

549 polynomial fits over the respective combined (global) fit variance. If the two variances are

550 close then the pre and post curves are almost identical suggesting a minimal training effect.

551 On the other hand, if the variance of the individual curves is greater than the combined

552 variance then the two curves are distinct indicating a possible training effect on the torque

553 output or voluntary activation.

554

555 
558 Figure 1: Rows 1-2: Passive Torque - angle plots with superimposed stimulation (vertical

559 line) for eccentric (row 1) and concentric contractions (row 2) at $50 \%$ s, $200 \%$ s and $350 \%$ 560 (columns 1-3 respectively). Rows 3-4: MVC (broken red line) and STIM (blue line) Torque 561 - angle plots with superimposed stimulation (vertical line) for eccentric (row 3) and 562 concentric contractions (row 4 ) at $50 \%$ s, 200\% and $350 \%$ (columns $1-3$ respectively). Black 563 broken line shows increasing/decreasing value of joint angle. All plots correspond to the 564 respective isovelocity regions.

565

566 Figure 2: Example plots from Subject 4 of the pre- and post-training T- $\omega$ raw data and 567 separately fitted MVT function for each dataset. The fitted MVT function produced maximal 568 concentric angular velocity values, $\omega$, of $1,550 \%$ s and $1,805^{\circ} / \mathrm{s}$ for pre and post-training fits 569 respectively. Those values compare very well with the values obtained by Forrester et al., 2011 for three different subjects $\left(1,410-2,000^{\circ} / \mathrm{s}\right)$.

571

572 Figure 3: Example plots from Subject 1 of the pre- and post-training \%VA- $\omega$ data and 573 separately fitted $3^{\text {rd }}$ degree polynomials for each dataset.

574

575 Figure 4: MVC (broken red line) and STIM (blue line) Torque - angle plots with superimposed stimulation (vertical line) during isometric contraction followed by passive 577 twitch.

578 Equation 1:

$$
\% \mathrm{VA}=\left(1-\frac{\text { superimposed twitch }}{\text { control twitch evoked at rest }}\right) \times 100
$$



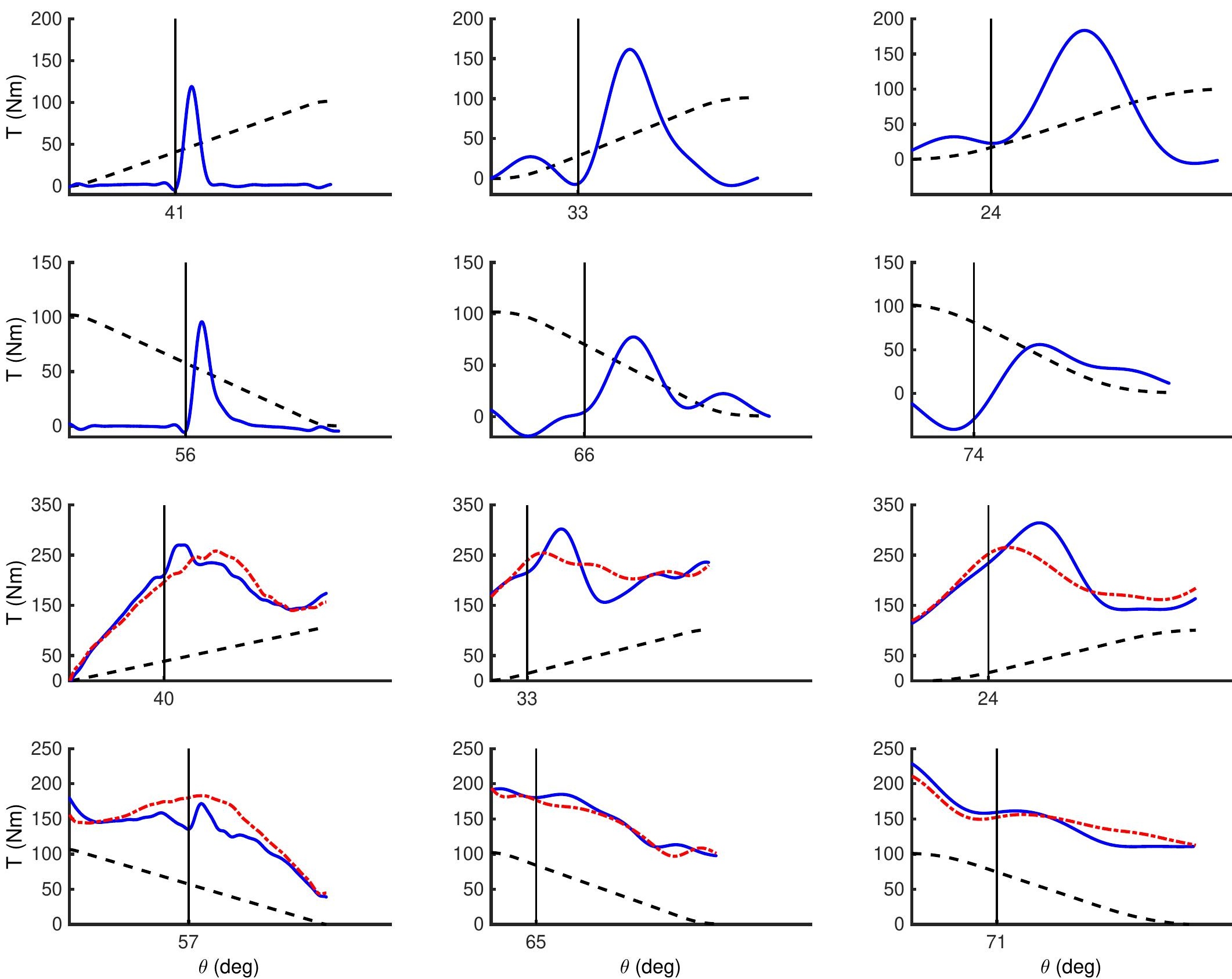


\section{Figure}

Click here to download Figure: Figure 2.eps

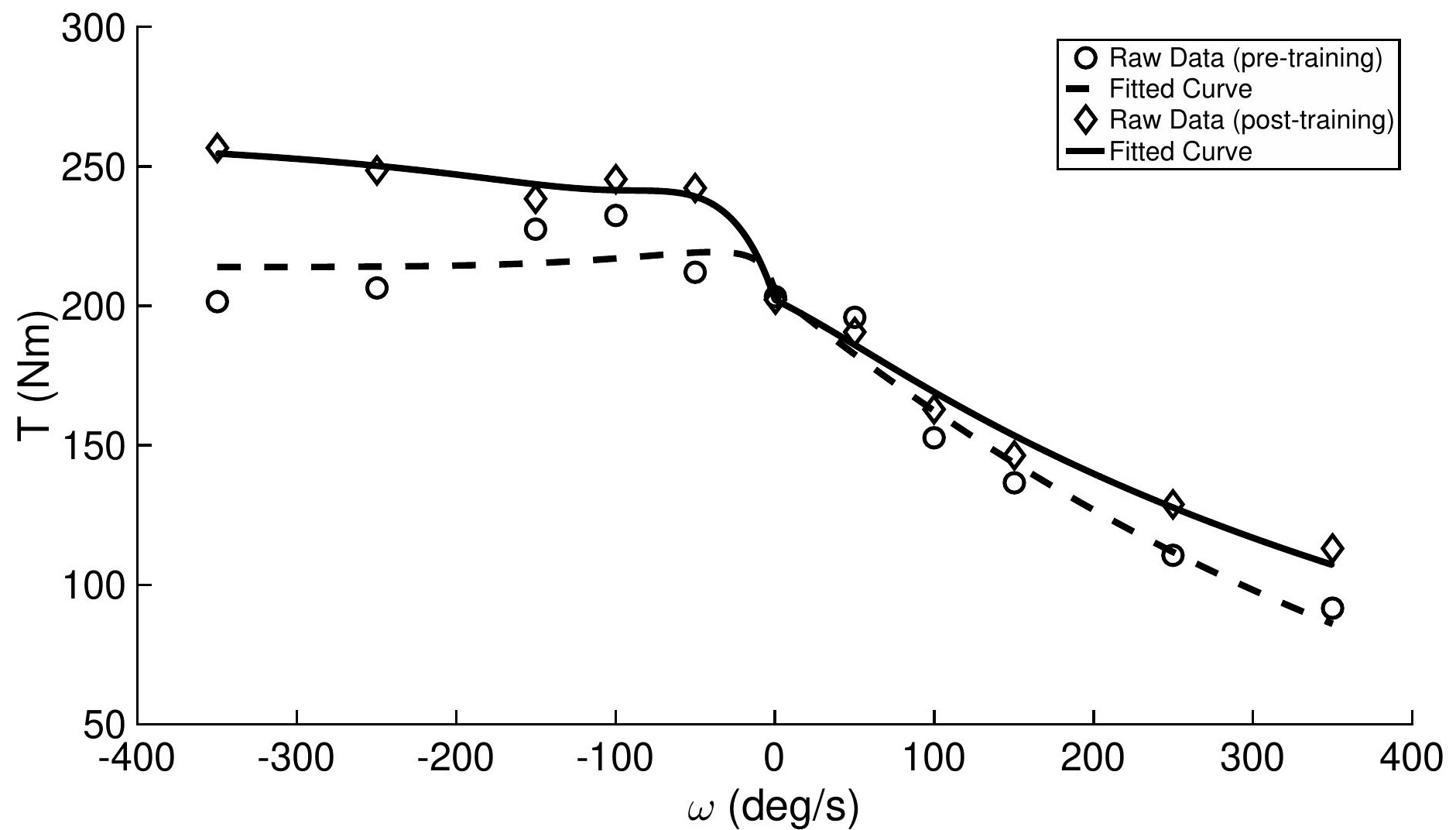

O Raw Data (pre-training)

Fitted Curve

Raw Data (post-training)

Fitted Curve 


\section{Figure}

Click here to download Figure: Figure 3.eps

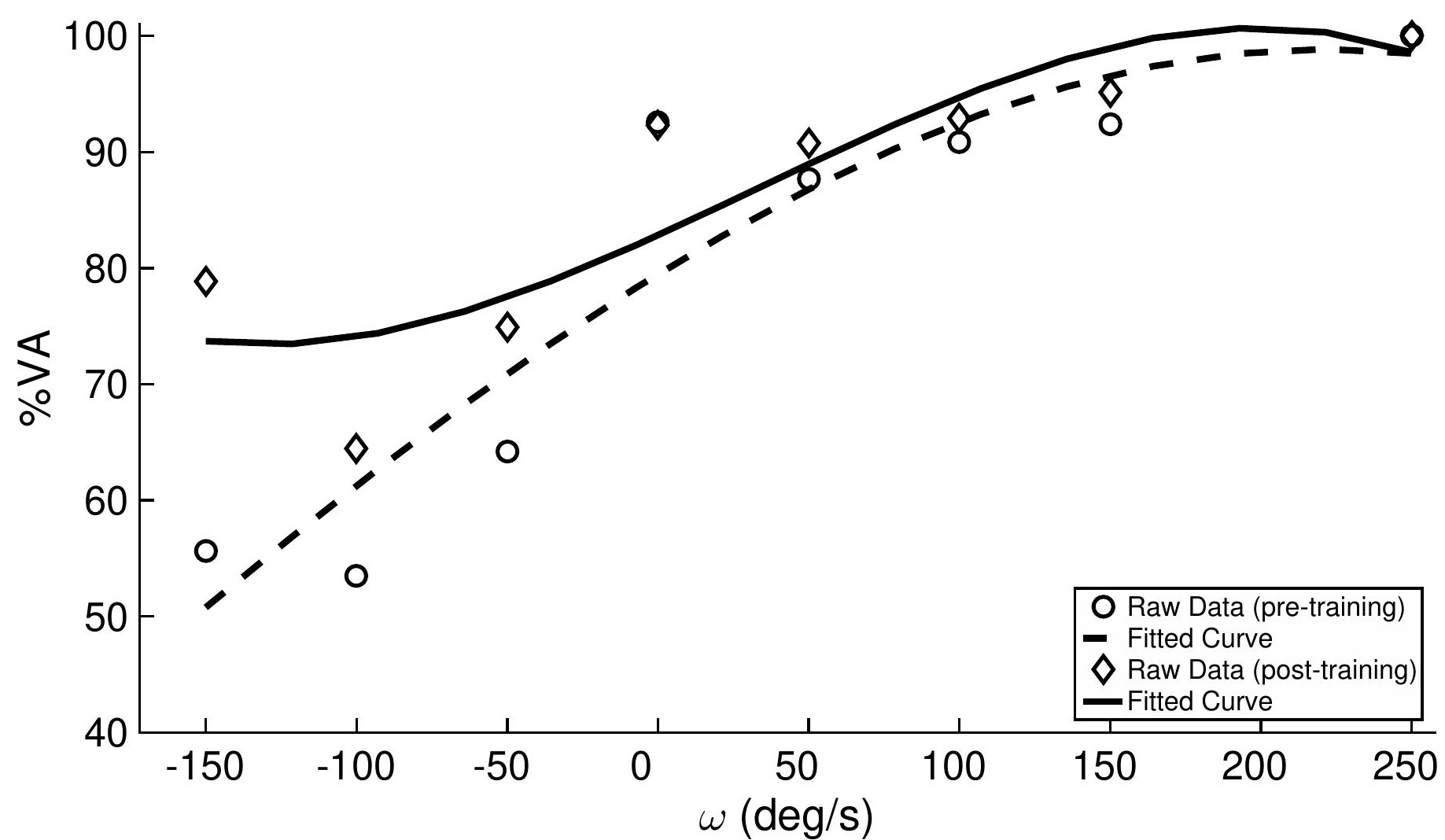

O Raw Data (pre-training)

- Fitted Curve

$\checkmark$ Raw Data (post-training)

$\omega(\mathrm{deg} / \mathrm{s})$ 


\section{Figure}

Click here to download Figure: Figure 4.eps

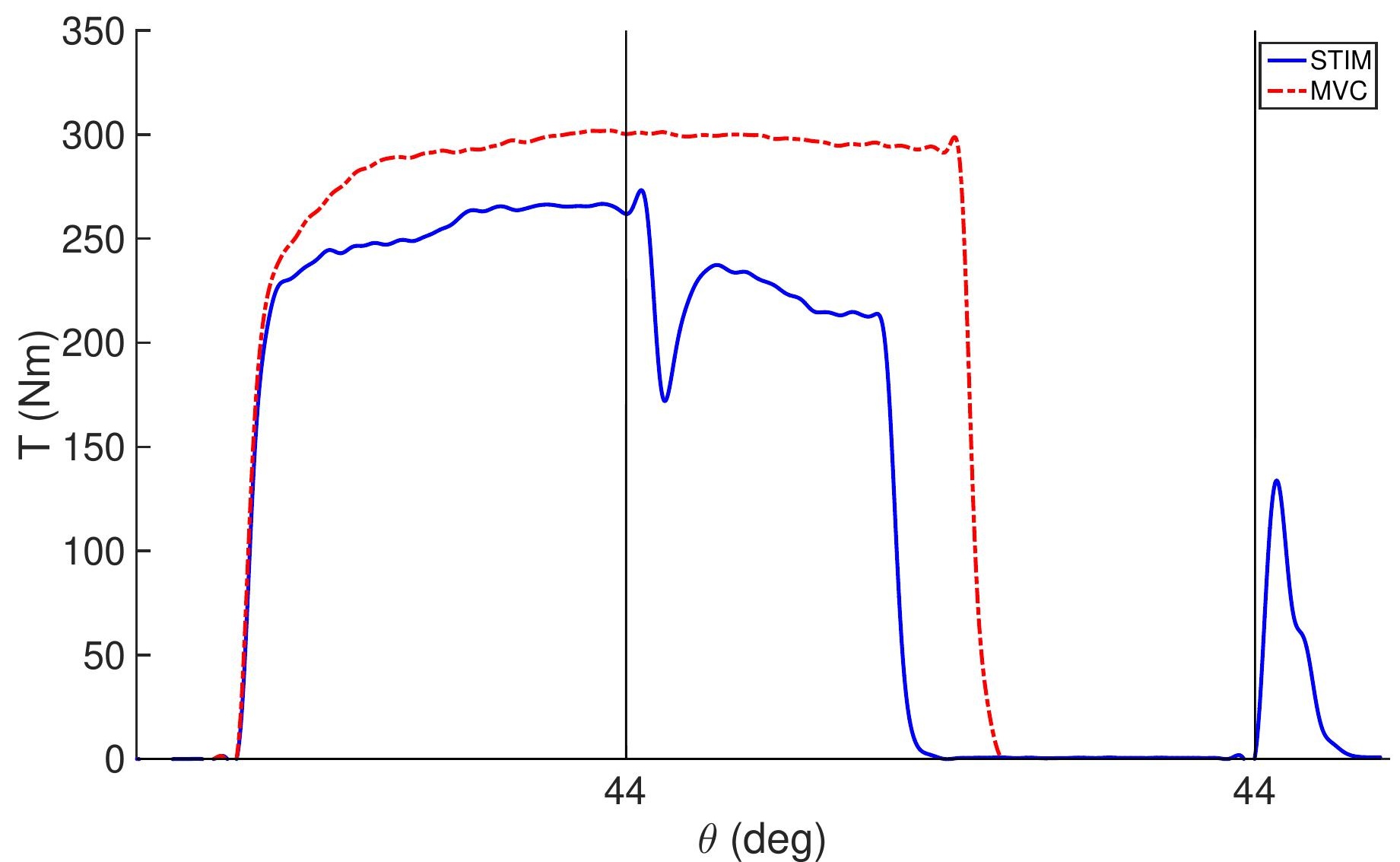

